\section{PTH-054 ERCP IN PATIENTS WITH MILDLY RAISED ALKALINE PHOSPHATASE AND NORMAL BILIARY IMAGING}

doi:10.1136/gutjnl-2013-304907.541

1."S Ghazanfar, 'S Qureshi. 'Surgery, Dow University Of Health Sciences, Karachi, Pakistan

Introduction Serum alkaline phosphatase levels in adults range between 20 and $120 \mathrm{U} / \mathrm{L}$. When bone disease is excluded, an elevation suggests biliary obstruction, injury to the bile duct epithelium, or cholestasis.

The mechanism for an elevated alkaline phosphatase has been related to enhanced synthesis and to release from cell membranes by the detergent action of retained bile salts. When there is partial biliary obstruction, the alkaline phosphatase will be elevated but the patient may not itch and the serum bilirubin will be normal. In high-grade total biliary obstruction, jaundice and itching will also be present. However, the significance of an isolated mild elevation of alkaline phosphatase (less than 1.5 to 2 times the upper limits of normal) has undergone only limited investigation.

Only a few studies have investigated the significance of a mild, isolated elevation of alkaline phosphatase. Because 1\% to $9 \%$ of people without symptoms have elevated liver enzymes, extensive evaluation of all abnormal test results would expose many patients to undue risks and expenses. On the other hand, failure to evaluate minor liver enzyme elevations could mean missing the early diagnosis of potentially treatable disorders. keeping this in mind we decided to look at the ERCP findings in patients with raised alkaline phosphatase levels in patients with cholelithiasis but with normal CBD status on imaging.

Methods A retrospective descriptive study was conducted at Surgical Unit 4 of Civil Hospital Karachi, over a period of 5 yrs, from August 2006 to July 2012. Sixty five patients with altered LFT's in terms of raised alkaline phosphatase and bilirubin and normal biliary tract on imaging were included in the study. Informed consent was taken from all patients and permission from hospital ethical committee was sought. All patients underwent standard ERCPs. And the findings and clinical data were entered on the special ercp database. Results were analysed using spss version 19

Results A total of 65 patients were included in the study. Mean age of study population was: $42.75+13.84$ (20-75 yrs). Mean bilirubin level was :1.04 + $1.03(00.0-4.59)$. Mean alkaline phosphatase : $168.98+73.259(110-714)$

Ercp findings:

Normal 50 pts $(76.9 \%)$

Stones 10 pts $(15.4 \%)$

Stricture 1 pt $(1.5 \%)$

Failed ercps: 4 pts (6.2\%) subsequently lost to followup

Successful duct clearance was achieved in 9 pts $(13.8 \%)$

stents were placed in 2 pts $(3.1 \%)$

Conclusion A minimally raised alkaline phosphatase may be associated with biliary obstruction as $15.4 \%$ patients in this study had choledocholithiasis

Disclosure of Interest None Declared.

\section{PTH-055 TO EVALUATE THE FREQUENCY AND RISK FACTORS IN THE POST ERCP PANCREATITIS WHERE ERCP ACCESSORIES ARE REUSED}

doi:10.1136/gutjnl-2013-304907.542

1,"S Ghazanfar, ${ }^{2}$ S Qureshi. 'Surgery, Dow University Of Health Sciences, ' 2 Surgery, Doew University of Helath Sciences, Karachi, Pakistan

Introduction Despite all the advances and improvement in ERCP techniques, post ERCP acute pancreatitis remains the most feared and common complication, being associated with high morbidity and mortality. The figures cited in literature vary from $1-7 \%$ to
$12-31 \%$. The pathogenesis of this condition is multifactorial and the severity of the condition may range from mild to severe pancreatitis. Most of the cases are of mild pancreatitis which usually resolves spontaneously without serious sequelae. The incidence of severe pancreatitis ranges from $0.3 \%$ to $0.6 \%$

The objective of our study was to look at the frequency of post ERCP pancreatitis in a setup, where ERCP accessories are reused because majority of the patients cannot afford the cost of new accessories for the procedure. Another objective was to look at the factors responsible for acute pancreatitis.

Methods This study was conducted at the endoscopy suite of surgical unit IV, Civil Hospital Karachi over a period of one year from $1^{\text {st }}$ December 2010 to $30^{\text {th }}$ November, 2011.

A total of 500 patients were included in the study. Patients who presented with pancreatitis or raised amylase levels pre procedure or patients who had previous history of surgery on the biliary or pancreatic systems were excluded from the study. Pearson Chi square and Fisher's exact test were used for qualitative data and t-test for quantitative data. Significance was taken as $p \leq 0.05$. Odds ratio was calculated for the qualitative data using $95 \%$ confidence interval.

Results Age of the study population ranged from 9 to 90 years (mean age 46.5 years \pm 14.94 , median 45 years). Male to female ratio was 1:1.87. Pancreatitis was seen in 18 patients (3.6\%). Mild in $15(3 \%)$, moderate in $1(0.2 \%)$ and severe in $2(0.4 \%)$. Mean amylase level at 4 hours and 24 hours was $280.93 \pm 539.13$ and $168.83 \pm 338.34$ respectively. Pancreatitis was seen in 15/326 (4.6\%) female and $3 / 174(1.72 \%)$ males. Statistically significant increased risk for pancreatitis was seen in difficult cannulation $(9.8 \%, p=0.006)$, prolonged cannulation time $(7.6 \mathrm{~min}, \mathrm{p}=0.002)$, pancreatic duct cannulation $(13.7 \%, p=0.001)$ and pancreatic duct contrast injection $(13.4 \%, p<0.001)$

Conclusion The frequency of pancreatitis was comparable to that of international literature. Difficult cannulation, pancreatic duct cannulation, pancreatic duct contrast injection and balloon sphincteroplasty increases the incidence of post ERCP pancreatitis. Reuse of ERCP accessories poses no additional risk to the frequency of pancreatitis.

Disclosure of Interest None Declared.

\section{PTH-056 DO DIABETIC PATIENTS HAVE ANY WORSE OUTCOMES THAN NON-DIABETIC PATIENTS AT COLONOSCOPY WITHIN THE BOWEL CANCER SCREENING PROGRAMME? A CASE CONTROLLED STUDY}

doi:10.1136/gutjnl-2013-304907.543

1,*S McGowan, 'B Moreton, 'E Simmons, 'E Smith, 'V Jackson, 'P Bliss, ${ }^{2} \mathrm{~S}$ Sarkar.

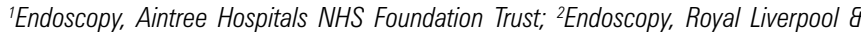
Broadgreen University Hospital, Liverpool, UK

Introduction Diabetic patients (DM) often have numerous comobidities and can suffer from autonomic dysfunction and poor GI motility. It is unknown whether their outcomes are any worse within the Bowel Cancer Screening Programme (BCSP) than NonDiabetic patients (NDM)

Methods An audit was performed in 2011 on 100 consecutive Diabetic (DM) and Non-Diabetic (NDM) patients that had received Moviprep as an oral cleansing agent (50 each group); in order to evaluate the quality colonoscopy outcomes within the Merseyside \& North Cheshire BCSP.

Results The mean age was 67.96 in DM and 67.44 in NDM groups. There were more males in the DM group (78\% v 54\%). Median ASA in both groups was 2 . The bowel preparation was poor in $14 \%$ of DM which led repeat colonoscopy in 7 patients. In the NDM poor bowel preparation was in $8 \%$ with 4 repeat colonoscopies. The caecal intubation rate (CIR) was $92 \%$ in each group but in the DM group the reason for failure was poor prep, whilst in the NDM is was acute angulation and sigmoid looping. i.e. not poor bowel 